\section{COGENERATION UNIT NOISE REDUCTION BY ITS CASE}

RADEK STRAMBERSKY ${ }^{1}$, VACLAV PAVELKA ${ }^{1}$, TOMAS PAWLENKA $^{1,2}$, PAVEL SURANEK ${ }^{1}$, LESZEK MORZYNSKI ${ }^{3}$

${ }^{1}$ Department of Control Systems and Instrumentations, VSBTechnical University of Ostrava, Ostrava, Czech Republic ${ }^{2}$ Department of Materials and Technologies for Vehicles, VSB-Technical University of Ostrava, Ostrava, Czech Republic

${ }^{3}$ The Department of Vibroacoustic Hazards, Centra Institute for Labour Protection - National Research Institute, Warsaw, Poland

DOI: 10.17973/MMSJ.2021_6_2020070 e-mail: radek.strambersky@vsb.cz

This paper deals with cogeneration unit noise measurement by the acoustic camera. Noise is not only measured as the final number of sound power levels, but also its original location is determined with the use of the beamforming algorithm. The properties of the used microphone array are considered and numerically calculated as every different microphone array layout will measure with another resolution. From the frequency spectrum, the possible technical source is determined. The results of noise source visualization show the cogeneration unit case noise decreasing effect while also offering the possibilities for design improvements.

KEYWORDS

Cogeneration unit, noise, acoustic camera, beamforming, noise measurement

\section{INTRODUCTION}

Localisation of noise sources is a crucial practice in the design and maintenance of machines because the requirement for modern tools and devices is to operate precisely and quietly. Noise is neither healthy nor ergonomic, and vibrating parts lead to malfunctions. The noise limits for different applications are decreasing every year. Another reason for the importance of acoustic noise diagnostics is that the noise spectra can be used as a tool for malfunction detection or life prediction. Vibrating mechanical parts, impacts, imbalance, malfunctions, or flow of air or other fluids are usually the sources of noise. For complex structures, the problem can arise from too many possible sources.

Exterior noise from the devices can usually be decreased by separating the excitation parts (e.g. an engine) by silent blocks, covering the noise area, or implementing active or passive vibration damping [Wrona, 2019]. For complex machines, it is a challenging task to perform a noise diagnostic without proper feedback.

The ideal tool for best understanding the condition of the device is the visualisation of noise. For this purpose, we can use acoustic cameras which allow us to see the sound power in the real image. However, for interpreting the results, it is mandatory to understand the method and behaviour on the measured frequency area as every camera will perform differently on various frequency bands. In this article, we use an acoustic camera to evaluate the construction of new cogeneration unit chassis and localise the noisiest sources of remaining noise for future innovations [Tuma, 2012].

\section{ACOUSTIC CAMERA METHODOLOGY}

An acoustic image or video consists of combining a pressure contribution or an acoustic intensity map with a digital picture. The idea is based on simultaneously measuring many acoustic signals, calculating the acoustic intensity power at specified points, and combining this intensity power map with a picture or recorded video. The sound power loss due to distance must be considered, when estimating the correct sound power. Otherwise, we are working with the acoustic pressure or intensity contribution at a measured distance.

The beamforming filter enables the calculation of the acoustic contribution at the specified point for far-field measurements. Near-field holography measurement methods use the information regarding the shape of the wave near the noise emitter. This method is not used for the diagnostics in this article. For every measured signal and every measured point, the aim is to estimate acoustic intensity, the times it takes to arrive from the measured point to the microphones are calculated. Then we delay the signals by these times to get the synchronised signals in the measured point. Lastly, we calculate the arithmetic average. Signals from the direction of the point are close to the original level, while all other directions are dampened. The algorithm is simple but demands many resources. For lower precision error due to discrete delay, a higher sample rate, signal resampling, and interpolation can be used [Gerges, 2009; Miljko, 2011].

The frequency range, in which we are still able to recognise the correct noise sources, depends on the microphone array pattern. Choosing the right criterion to compare different patterns can be difficult. For this article, we are using the Bruel\&Kjær WA1764-W-001 acoustic camera [Bruel \& Kjær 2020]. The camera uses their patented optimising method by lowering the MSL (maximum sidelobe) where the mainlobe is in the direction of the real noise and the sidelobes are in wrong directions [Hald, 2004a]. The camera's 30 microphones are specially designed, being built into the microphone array enabling good results for near-field and far-field localisation, while keeping the dimension of the camera small. Randomised patterns show favourable results with far-field localisation while organised patterns (e.g. grids) perform well with near-field localisation. The design of this microphone grid combines these two approaches by dividing the camera into the same segments while optimising the microphone positions in the segment numerically by minifying the MSL.

For correct interpretation of the measurement, it is mandatory to understand the method limits of the camera used as every microphone array will perform differently for contrasting frequency bands. We were not able to use beamforming for low frequencies up to $1,5 \mathrm{kHz}$ due to the results being unclear, making it hard to find the correct location of the noise. However, for these low frequencies, the acoustic camera can be used with the near-field holography SONAH algorithm. The directional characteristic for $1.5 \mathrm{kHz}$ can be seen in Figure 1 .

With a higher frequency, the resolution improves, as can be seen from Figure 2; however, more side lobes appear. These results were obtained by numerical simulation of a simple beamforming algorithm with the given microphone pattern. The performance (MSL) then increases with the frequency of measured noise (more and stronger side lobes appear). 


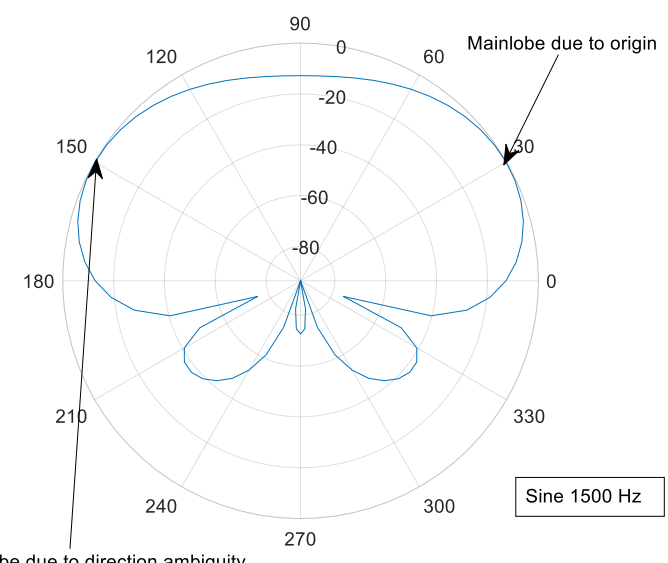

Opposite lobe due to direction ambiguity

Figure 1. Beamforming filter response for different angles in dB

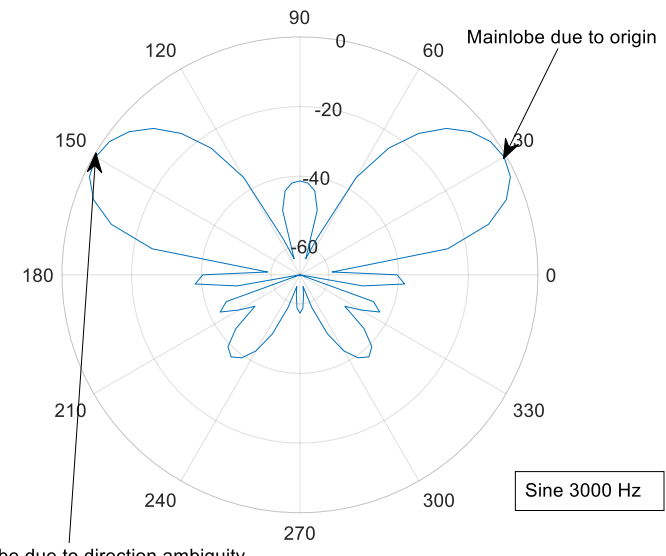

Opposite lobe due to direction ambiguity

Figure 2. Beamforming filter response for different angles in $\mathrm{dB}$

Bruel\&Kjær provide the MSL dependence on frequency in their product information (see Figure 3). Higher frequencies are hard to localise as MSL decreases, creating ghost images from erroneous locations. Practically, this array can localise noise sources up to $12 \mathrm{kHz}$. The MSL can be decreased with a higher sample rate or interpolation between samples, so the time delay can be calculated more precisely.

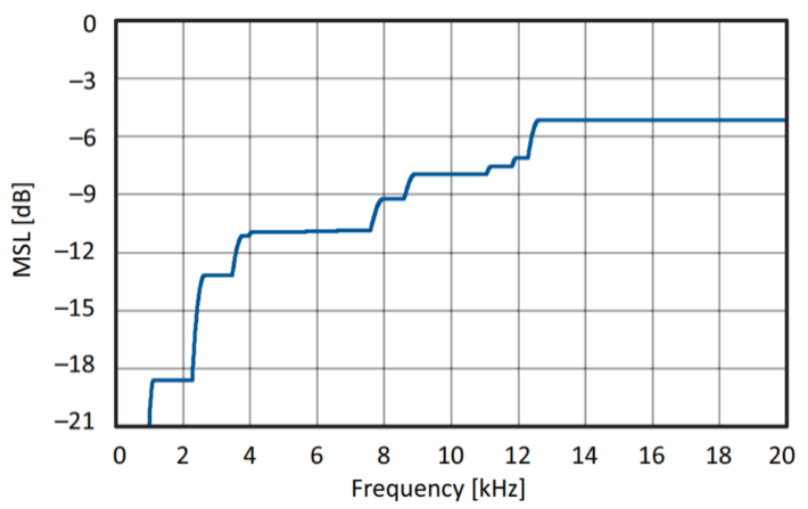

Figure 3. Frequency to MSL (Maximum Side Lobes) dependence [Hald, 2004a]

\section{COGENERATION UNIT DESIGN}

The cogeneration unit shown in Figure 4 is intended for the home accumulation of heat and electrical energy. Since this development is designed for use in ordinary households, there are increased requirements for low noise. The basis of the cogeneration unit is a petrol engine, which is the primary source of noise within the measured acoustic spectrum. Apart from the engine, there is also a gearbox. The output shaft is connected via a flexible coupling to an asynchronous generator. The cogeneration unit also includes two fans. One is used for the air inlet and cooling of the radiator. The second serves as an air outlet from the engine chamber.

The whole frame of the cogeneration unit is covered with a designed housing, which is lined with anti-noise foam from the inner side to reduce the overall noise. However, the housing contains openings for air inlet and outlet.
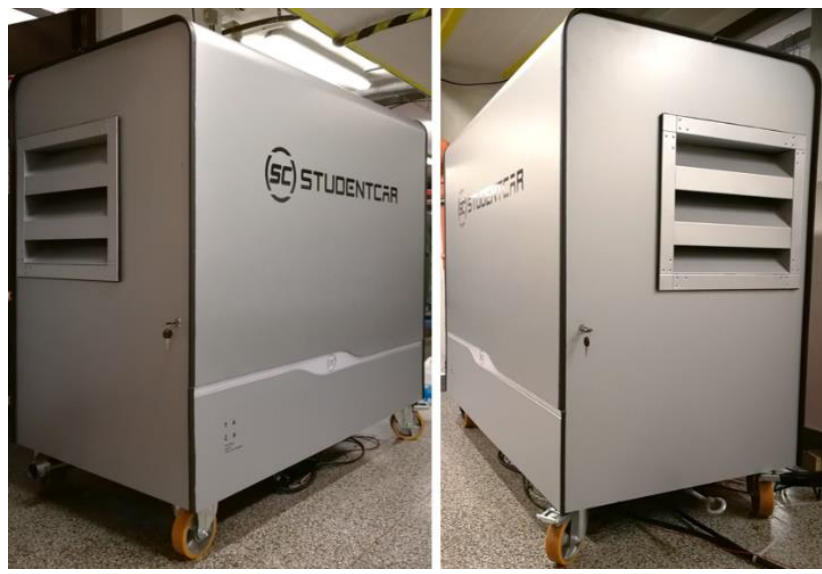

Figure 4. Cogeneration unit

Figure 5 includes a description of the cogeneration unit inner components, which are involved in the acoustic noise of the complete unit.

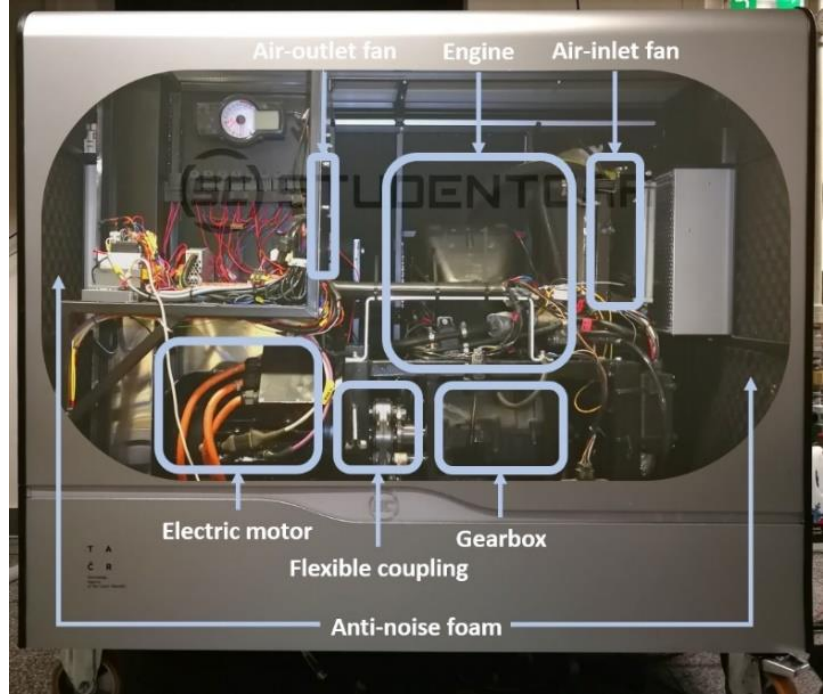

Figure 5. Cogeneration unit inner components

Not only are the mechanical components significant noise sources but also the exhaust pipeline, although they were equipped with two exhaust silencers to reduce noise. The noise can also get through the unit's bottom section, through which the cables and the gas hose are routed. The operating range of the cogeneration unit engine speed is from 3200 RPM to 6000 RPM, which corresponds to the output shaft speed from 1350 RPM to 2500 RPM.

\section{MEASUREMENT}

The main goal of the measurement was to compare the effect of reducing the sound pressure level of noise made by the designed cogeneration unit case. Due to the cogeneration unit system connections the noise measurement was taken from the inner 
space with the limitation of noise reflection. The conditions were the same for all measurements; thus, these results are compared. We had three basic types of monitored operating conditions: idle speed, increased speed of 3500 RPM without load, and increased speed of 3500 RPM with load. All these measurements were repeated with and without a running second fan installed inside and all these written combinations were measured with an open and closed case to compare the differences and its efficiency of reducing noise. The measurement was taken by an acoustic camera with an outer diameter of $300 \mathrm{~mm}$ using 30 channels at a distance $1.2 \mathrm{~m}$ from the cogeneration unit. As seen in Figure 6, the measurement was carried out from two positions, one after another. We made multiple measurements with different conditions and then calculated the noise difference based on altered properties (opened/closed case, load, fans).

The measured signals are weighted by an A-type acoustic filter, which reduces the lower frequency to correspond to the human hearing. As explained before, the measured range for the used camera used for far-field localisation is above $1500 \mathrm{~Hz}$. Therefore, this filtering does not significantly affect the results.

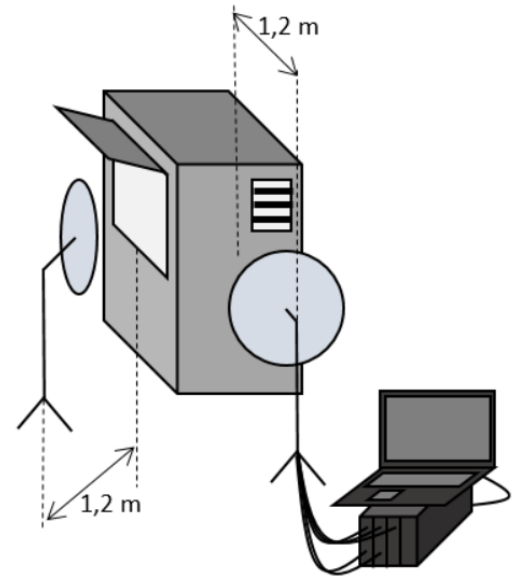

Figure 6. Measurement scheme

\section{RESULTS}

From the measured data, it is clear to see that there is almost zero effect of turning the fan on and off. The noise from the combustion engine exceeds the fan noise. Furthermore, it was noticed that adding the load at 3500 RPM takes effect in increasing the noise power covered by the camera image by approximately $1,5 \mathrm{~dB}$. A change of RPM naturally raises the noise by $7,4 \mathrm{~dB}$ on average. The most researched factor-cogeneration unit case noise damping efficiency - is confirmed by the absorption of $14 \mathrm{~dB}$. Differences in acoustic camera position during the experiment (measurements from the front and the side) are not significant. All results are recorded in Table 1.

\begin{tabular}{|c|c|}
\hline Conditions & AVG $\Delta \mathrm{L}_{\mathrm{w}}[\mathrm{dB}]$ \\
\hline Fan added & 0,38 \\
\hline Load added & 1,48 \\
\hline Increasing of working speed & 7,38 \\
\hline Case open & 14,10 \\
\hline $\begin{array}{c}\text { Measuring from the front vs } \\
\text { from side }\end{array}$ & 1,36 \\
\hline
\end{tabular}

Table 1. Measurement results

The average $\Delta \mathrm{L}_{\mathrm{w}}$ represents the average sound power level covered by the camera. The average is calculated from six different measurements.

The loudest noise was obtained by measuring 3500 RPM with an open case, load and working fan. For this case, we measured a sound power of $85 \mathrm{~dB}$. With the case closed, it reaches approximately $70 \mathrm{~dB}$.

The strongest source of the noise is the gearbox, which is usually the field of research for noise reduction, as seen in Figure 7. The reason is the impact of the gears' contacts. The second expected source of noise is the combustion engine. However, as seen from the measurements, the noise from the ignitions are lower than the gearbox. Other components are silent compared to these two dominant sound sources.

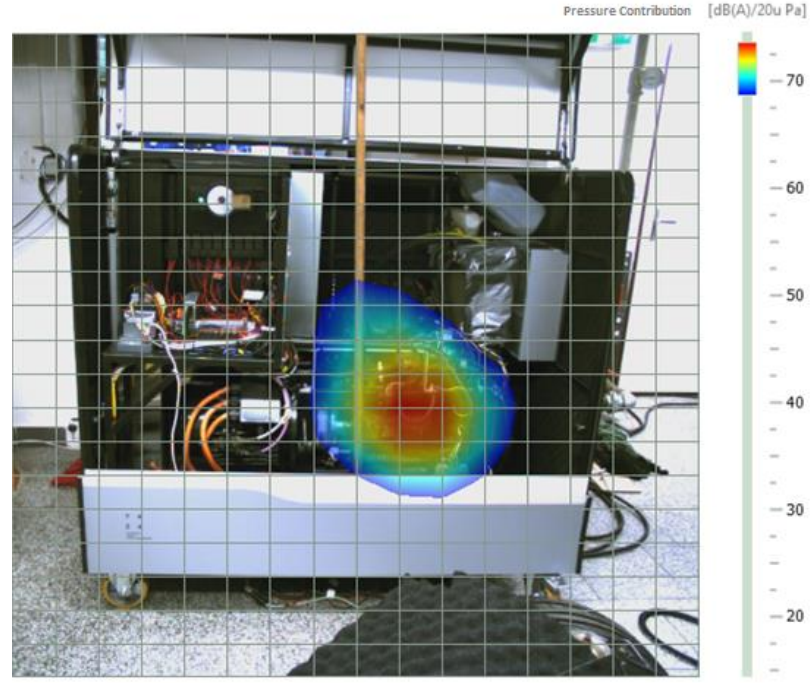

Figure 7. Visualisation of noise sources of the cogeneration unit using the acoustic camera - opened case, front view.

The newly developed case for this cogeneration unit is much more powerful in terms of noise reduction and represents a measured difference of $14 \mathrm{~dB}$ in the sound power covered by the camera image. The weak points are the areas which are not enclosed and are used for air ventilation. To be more specific, the remaining noise comes from the bottom of the unit and the ventilation hole as can be seen in Figures 8 and 9 .

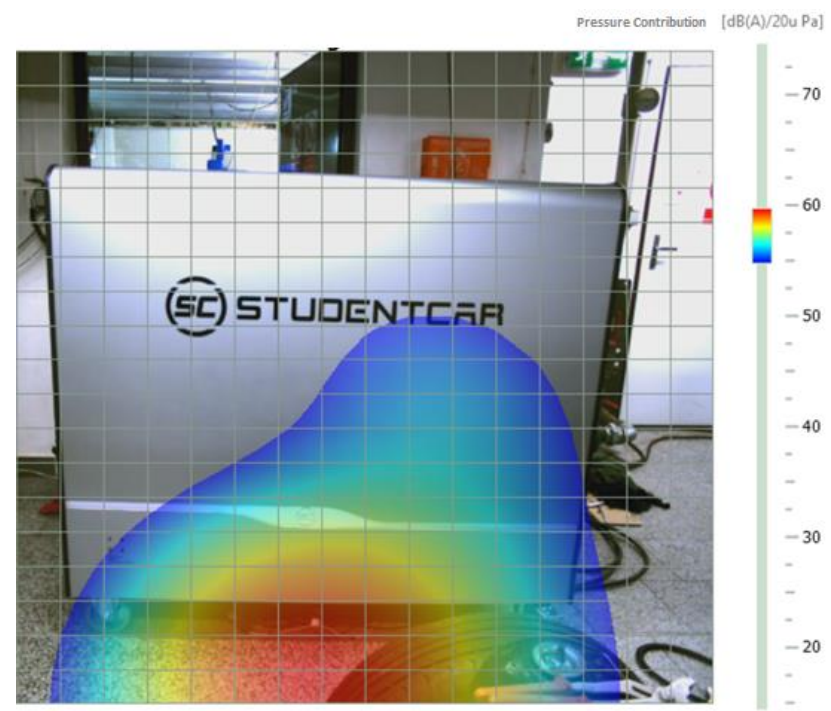

Figure 8. Visualisation of noise sources of the cogeneration unit using the acoustic camera - closed case, front view. 
Although noise leaking through the ventilation hole is difficult to reduce due to the airflow needed, there are still possibilities to reduce the noise by properly designing the bottom part of the unit case while also considering all connections.

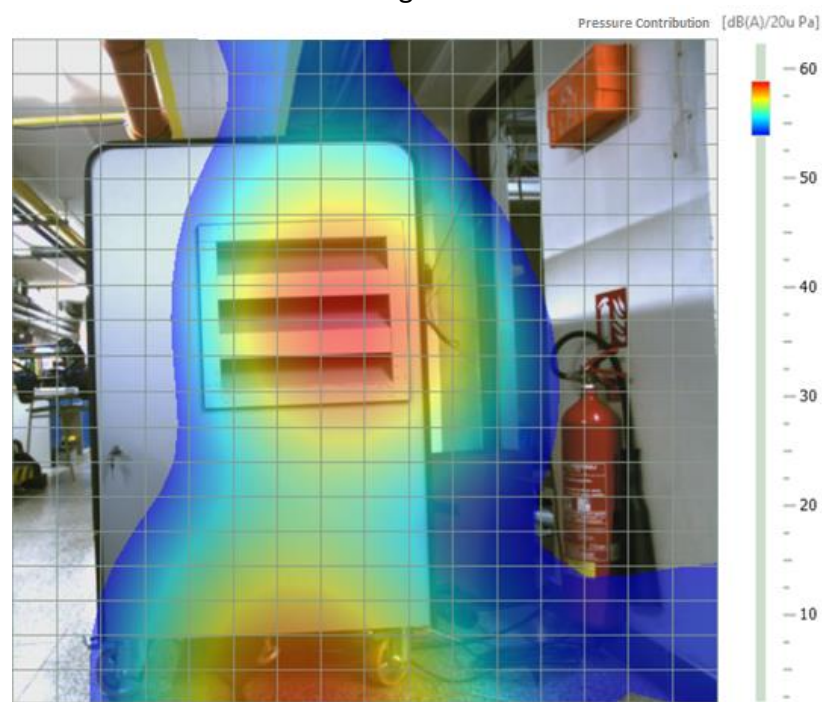

Figure 9. Visualisation of noise sources of the cogeneration unit using the acoustic camera - closed case, side view

\section{CONCLUSION AND DISCUSSION}

This paper is focused on the reduction of cogeneration unit noise made by its case. Measurement was realised by using an acoustic camera. From the results provided in Table 1, we can see the effect of decreasing the sound power of noise made by the cogeneration unit case by $14 \mathrm{~dB}$ on average for the measured difference of closing the case from $85 \mathrm{~dB}$ to $70 \mathrm{~dB}$. The sources were determined, and it has also been clarified where the remaining noise is coming from for future inventions.

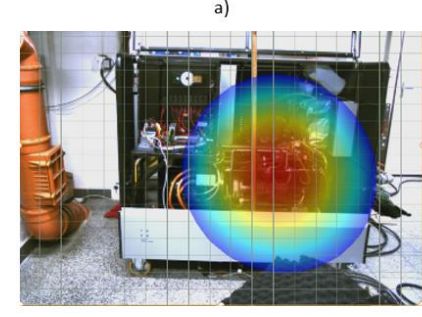

c)

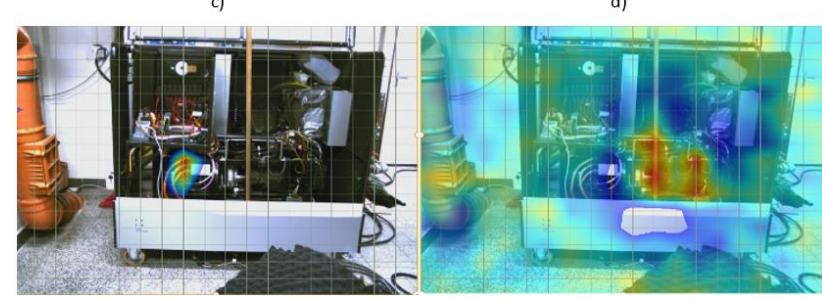

Figure 10. Visualisation of microphone array behaviour in different frequency bands: a) $1500-3000 \mathrm{~Hz}$, b) $7500-9000 \mathrm{~Hz}$, c) $15000-16500$, d) $18500-20000 \mathrm{~Hz}$

In Figure 10, the features of the camera which were described in Chapter 3 can be seen. In low-frequency bands, we can see that the source cannot be determined very accurately. With a higher frequency (Figure 10b), the noise source becomes more precise. While the measured frequency range in Figure $10 \mathrm{c}$ is out of the recommended range [Bruel \& Kjær, 2020], we get more accurate localisation of high-frequency noise made by an electrical generator. The results are usable owing to these noises being the only source on this band. MSL is still small enough in this picture.
Even with a higher frequency (Figure 10d), we are still able to see the source (gearbox), but the image contains many ghost images which are created not by a reflection but from the very low MSL. These acoustic camera properties are always need to be considered when the evaluation is done.

\section{ACKNOWLEDGEMENTS}

This work was supported by the European Regional Development Fund in the Research Centre of Advanced Mechatronic Systems project, CZ.02.1.01/0.0/0.0/16_019/ 0000867 within the Operational Programme Research, Development and Education and the project SP2021/27 Advanced methods and technologies in the field of machine and process control supported by the Ministry of Education, Youth and Sports, Czech Republic, and research task (II.PB.20) carried out within the National Programme "Improvement of safety and working conditions" partly supported in Poland in 2020-2022 within the scope of research and development - by the Ministry of Science and Higher Education/National Centre for Research and Development and in the framework of the grant programme "Support for Science and Research in the Moravia-Silesia Region 2018" (RRC/10/2018), financed from the budget of the Moravian-Silesian Region.

\section{REFERENCES}

[Bruel \& Kjær 2020] Bruel \& Kjær. "PRODUCT DATA. BK Connect Acoustic Camera Type 9712-W-FEN". Nærum, Denmark, BP 2534 - 18 2020-06.

[Gerges 2009] Gerges, S. N. Y., Fonseca, W. D. (2009, July). " State of the Art beamforming Software and Hardware for Applications ". In Proceedings of the 16th International Congress on Sound and Vibration (17 p.).

[Hald 2004a] Hald, Jorgen. "Combined NAH and beamforming using the same microphone array." Sound and Vibration 38.12 (2004): 18-27.

[Hald 2004b] Hald, Jorgen. "Beamforming", Bruel \& Kjaer Technical Review, nr.1 2004.

[Miljko 2011] Miljko, M. E. "Some Research Challenges of Acoustic Camera"., ". In Proceedings of the 19th Telecommunications forum TELFOR 2011 (4 p.).

[Tuma 2012] Tuma, J., Janecka, P., Vala, M., \& Richter, L. (2012, May). "Sound source localization". In Proceedings of the 13th International Carpathian Control Conference (ICCC) (pp. 740743). IEEE.

[Wrona 2019] Wrona, S., Mazur, K., Rzepecki, J., Chraponska, A. Pawelczyk, M. (2019, July). Reduction of compressor noise by the active casing approach. In Proceedings of the 26th International Congress on Sound and Vibration (7 p.).

\section{CONTACTS:}

Ing. Radek Strambersky

Department of Control Systems and Instrumentation, VSB-Technical University of Ostrava 17. listopadu 2172/15, Ostrava, 70800 Czech Republic radek.strambersky@vsb.cz 